\title{
Village Level Information System - A Tool for Decentralized Planning at District Level in India
}

\author{
J. Adinarayana ${ }^{1 *}$, F. J. Raj ${ }^{1}$ and V. Sharma ${ }^{2}$ \\ ${ }^{1}$ Centre of Studies in Resources Engineering, Indian Institute of Technology-Bombay, Powai, Mumbai 400 076, India \\ ${ }^{2}$ National Informatics Centre, Ministry of Communication and Information Technology, A-Block, CGO Complex, Lodhi Road,
} New Delhi 110 003, India

\begin{abstract}
Geographical Information Systems and the underlying Spatial Data Infrastructures have potential to assist in planning, monitoring and exchange of information between various agencies in Rural Development administration. The paper describes the present decision making process and the rural informatics situation in Indian districts. A study was undertaken in a tribal-oriented and rural district in India to demonstrate the integration of village-level spatial and non-spatial data into a useful tool, called 'VLIS' (Village-Level Information System), for decentralized planning. This simple and robust tool will assist the decision-makers to generate various ecological and socio-economic views for identifying candidate villages for rural watershed management schemes (prescriptive and executive level planning). The paper also envisages future development and usefulness of this community-level GIS tool for grass-root planning.
\end{abstract}

Keywords: Decentralized planning, decision-making tool, geographical information systems, rural development, spatial data infrastructure, village-level information system

\section{Introduction}

In recent years, in India, there has been a blossoming of participatory planning at local levels fostered by NGOs, but the established procedure for rural development planning remains top-down, by way of schemes to address specific problems and opportunities. These schemes are mandated and financed by the state or central government and implemented by sectoral institutions in the district involved in rural development planning. Some of the specific items for attention drawn up by the $11^{\text {th }}$ schedule, $73^{\text {rd }}$ and $74^{\text {th }}$ Constitutional Amendment Acts, 1992 of India are: Land improvement, implementation of land reforms, land consolidation; Soil Conservation; Minor irrigation, water management, watershed development; Social forestry and farm forestry; Roads, culverts, bridges, ferries, waterways; Drinking water; Fuel and fodder; Rural electrification; Adult and non-formal education; Poverty alleviation programs, etc.

Due to prescriptive form of land use planning in the districts and an urgent demand to improve the management and delivery of the services, different and innovative approaches and systems/tools are required to solve the urgent problems facing these districts. These new methods are expected to assist in providing the required resources and management in a more efficient and effective manner. Unfortunately, many of the current methods and approaches in rural land use planning in the districts have proven only partly successful. Rural

\footnotetext{
* Corresponding author: adi@iitb.ac.in
}

development planning is also being decentralized to take account of local conditions and needs. Districts are now required to draw up and implement integrated development plans, and those districts that have independent tax-raising powers are in a position to take the initiative. This task is made more difficult by the sectoral structure of government and the scarcity of village/watershed level formal information about natural resources, social and economic conditions and infrastructure.

\subsection{Spatial Data Infrastructures for Rural Development at District/Sub-District Level}

Over the last decade, districts in developing countries enthusiastically embraced Geographical Information Systems (GIS) (applications) and the supporting spatial data infrastructures (SDI) (the geodetic control and common base maps) (Bishop et al., 2000). It is foreseen that where geographical aspects are important for rural management, decision-making and planning, these GISs and SDIs would help to improve the efficiency of these rural management activities.

Typically, more than $90 \%$ of information required for a district administration has a spatial component, such as parcels of land, road networks, infrastructure facilities, land use/cover, etc., with the result that GIS has been seen as an essential technology for rural management. Presently, in many of the districts such spatial information related activities are undertaken manually if at all. These districts are mostly larger in human resources, are rapidly expanding and hence are very anxious to adopt any technologies, which promise to make the 
management of these districts/villages more effective and efficient. However, institutional environment in these districts to allow the adoption of GIS is very different from the same administrative blocks in different parts of the country.

Presently, there is little or no spatial information infrastructure in the districts (particularly, large scale base maps). The biggest single barrier stopping the construction of a spatial infrastructure is lack of data. In many cases, the spatial data is in the form of un-scaled sketches. Where maps exist they are often out dated or classified as restricted information and access by public departments is very difficult if not impossible. Where current and unclassified maps exist, they are usually of different scales aggravating the problem of sharing information efficiently among various sectors of the district. Digital representation of spatial data is even rarer due to the lack of appropriate equipment and trained staff to establish and manage the infrastructure. Few districts, e.g. Kolar district in southern part of India with the help of Department of Science and Technology (DST), are ready to begin generating digital spatial databases as the task is seen to be too large, too costly and too complex both technically and administratively. Other limitations include inefficient processes for purchasing spatial information or GIS technology; and lack of funding or political will to support the construction of the infrastructure.

Development of a Spatial Decision Support System (SDSS) for decentralized planning using GIS technology is one of the important aspects to address this information problem (Adinarayana et al., 2000). The development of such SDIs and the resulting GIS, which can build upon them, are considered an essential requirement for the districts to improve their management. However, the tool has to provide a consistent level of information and analysis based on data that are available now, nation-wide, and this information has to be useful to today's decision makers without the need for specialist training. At present, the options are limited by the availability and scale of fundamental data (Table 1).

The data available for several of these fields are inadequate for many grass-root level decision-making processes that could otherwise be used to supply the information that decision-makers want. For instance, identifying the sites for mandated government sponsored poverty alleviation schemes. The parameters are simply not available from the existing maps of whatever scale and the census data. It is always possible to collect detailed data for specific attributes for special study areas, but this effort cannot be replicated in other districts with different ecological and socio-economic situations.

\subsection{Village Level SDIs for Rural Development}

A well-organized information system through the DISNIC programme of the National Informatics Centre (NIC), Ministry of Communication and Information Technology, Government of India (http://www.nic.in), has emerged in the country at district/sub-district level to facilitate development planning, responsive administration and promote informatics culture at the district/sub-district level. Its immediate clients are planners and the professional staff of various line ministries in the districts. Village wise digital database on census data, on both biophysical and social and economic dimensions, for most of the districts in India are available with NIC District Centers in different database formats. However, vil-

Table 1. Fundamental Data Availability in the Districts

- Digital 1:50,000 scale Survey of India topographic maps, contour interval $20 \mathrm{~m}$.

- An overlay of district and block boundaries with village centres identified as points.

- Social, economic and agricultural census data (e.g. proportion of irrigated land) held in tabular format by administrative unit.

- Agro climatic data, held in tabular format by point. There is an India Meteorological Department station in each district and a much more intensive network of rainfall stations. At a more generalized level, the country has been divided into agro-ecological zones that are matched with crop requirements.

- $\quad$ Geological Survey and, sometimes, geomorphological maps at 1:250,000.

- Land cover interpretation of 1:250,000 satellite imagery.

- All India Soil Survey maps at 1:250,000, sometimes at 1:100,000 and 1:50,000.

- Nation wide Census of India data of 1991 in digital form by the NIC. Latest 2001 Census data is available in some pockets from the NIC.

- Census GIS - an interactive thematic census data (of 2001) on demographic details online for district and state level. 
lage-based spatial data infrastructures are not available with the rural extension community in the districts for taking decisions on various rural development schemes.

In general, the rural development schemes are on a watershed basis. Each scheme is bounded by certain government policies, which have social, economic and biophysical dimensions. Policy is enshrined in their directives that establish the scheme and these commonly lay down the criteria for site selection. For example, to identify the National Watershed Development Program for Rainfed Agriculture (NWDPRA) watersheds, a centrally sponsored watershed management scheme, the decision-maker needs information on area of the watershed; and annual rainfall (by Theissen Polygon method), percentage-irrigated area, under-privileged (scheduled caste/ tribe $-\mathrm{SC} / \mathrm{ST}$ ) population of all villages falling in a watershed. Size of a watershed for this scheme is $\approx 10,000 \mathrm{ha}\left(100 \mathrm{~km}^{2}\right)$, and may not correspond to the physical geographer's concept of a natural watershed. Other schemes may include social factors such as poverty, under-privileged people (SC/ST), etc., and may consider smaller areas as primary watersheds. A problem with this decision method is that there is no standard watershed map. These are only delineated once they are identified. Also there is no standard method for delineation. Local field staff will recommend sub-watersheds, starting from the highest point, and preferring those with (1) a range of land types, so that many departments can be involved (e.g. territorial forestry, social forestry, agriculture, horticulture), (2) the most severe physical problems, and (3) the most severe social problems. Useful SDIs, in the form of Spatial DSS for rural land use planning (Adinarayana et al., 2000) and identification of priority sites for watermills in a watershed (Durga Rao et al., 2002), have been developed to implement in the government sponsored watershed manage- ment schemes. However, simple, low cost, project oriented, easily maintained and user-friendly spatial information technologies have the best chance of success (Bishop et al., 2000).

\section{Village Level Information System}

An attempt has been made in the present study to integrate spatial village maps with the NIC village-level census data to generate a GIS-based information system for grassroot level development planning, the prototype module of which will be called 'Village Level Information System' (VLIS). VLIS is broadly defined as a computer-based information system that assists the decision-maker to facilitate spatial planning for watershed/rural development programs at grassroot/village level. Primary objectives of the VLIS are:

1. to educate, train and promote informatics culture/experience to district officials;

2. to evaluate the need for appropriate common base map to be used by all Rural Development partners;

3. to design own management packages by the rural extension communities according to local needs and conditions.

VLIS will support the decision-makers to generate various views for developmental planning taking into considera- tion of local needs and constraints. An important part of the suggested approach on VLIS is to provide short term, useful and low cost outputs. These objectives were conceived on the basis that the GIS technology should integrate various agencies responsible for the rural development. Also, the technology should be appropriate to the needs and resources available in the districts, be relatively simple yet should be able to perform the task, be cost effective, and be application and user oriented.

It is expected that significant cost savings and an improvement in efficiency would result from sharing substantial core data sets and particularly the base map, since it was acknowledged that data acquisition would be the most important cost factor in developing GIS in the partner departments concerned with rural development.

The VLIS module has been developed for the tribaloriented and rural-based coastal district, called Thane of Maharashtra State in western India. Views for decentralized planning on rural development decisions are developed for rural-based Dahanu sub-division of the district, containing three talukas (sub-district or block): Palghar, Dahanu and Talasari.

\subsection{Data and GIS issues for $V L I S$}

Reproduction of maps, where they exist, is often done with ammonia printers with the original maps prepared and updated on transparent sheets. Cadastral data is usually stored in hard copy registers and updated manually where available. Where a digital database is being prepared, map production often continues to be done manually in parallel.

Often maps showing land ownership are in the form of sketches, which are not to scale. Therefore, the exact demarcation of the much of the land is impossible or very difficult and time consuming. Most information about land is in the experienced hands of key personnel in se- parate departments. Due to the absence of any other infor- mation system, they are treated as the primary information source. As a result of this, information can be easily lost.

Different parameters are available at different intervals. Usually, census details of the districts are updated for every 10 years. Some of the dynamic details, such as livestock census, etc., are available for every 4 to 5 years. Until now these data have not been linked to any spatial reference or mapping system and consequently could not be fully utilized for decision making. It is the view of some of the senior officials in the district that the mapping of these data after simple analyses will not only be very useful in assisting the management in rural systems but will also be very influential in promoting the wider use of GIS in the districts.

Using these data, set of maps can be produced to assist decision-making on various aspects of the rural management in the districts. Particularly, these spatial analyses will be very useful in their strategic planning such as 'annual plans'. The district officials are expected to be knowledgeable and have experience and expertise in the applications area for which they are responsible. They are more concerned with long term 
strategic planning for which maps at reasonable scale is sufficient.

In the present study, village maps were obtained from the local government agencies. Census data (of 1991) in digital format was procured from the District NIC office. Survey of India (SOI) topographical map (1:250,000 and 1:50,000 scale) and the District Census Hand Book (Anonymous, 1995) were also used as ancillary data. The attribute data used include many of the normal statistics collected in most districts by the Local Revenue Department and Census India such as demographic details, public facilities (schools, hospitals, banks, etc.), land use, and other environmental and socioeconomic dimensions.

The success of small strategic applications can help establish the value of GIS in the districts. For any rural development organizations in the districts, this VLIS experience suggests such a strategic application oriented approach is more relevant than a system-wide comprehensive strategy. This may not be the ideal from a system development point of view in the medium to long term, but it is the reality. It is recognized in the context of the present situation in the districts that it is very important that the cost and investment in GIS is recovered through short term benefits as usable products in rural land development. The cost benefit curve of an institutional GIS is steep i.e. the full benefits of GIS are realized often only in the long term, and in the intervening stages costs outweigh the immediate benefits to the community. In general, a district cannot produce GIS benefits without an investment in the underlying infrastructure.

There are a range of GIS implementation strategies in developed countries. Three main steps of planning, analysis and implementation are of importance in GIS implementation (Korte, 1994). The diffusion of GIS in an organization has been extensively discussed (Campbell and Masser, 1995) and the main phases established by the NCGIA (1990). The adoption of new spatial information technologies in developing countries should also be considered in the context of these main steps and approaches. However, these approaches should be modified by taking into account the following factors which can impede the adoption of spatial information technologies in developing countries : need for awareness and management support; data standards; data availability; personnel; resources; procedures; vendor support; successful implementation; and data integration (Bishop et al., 2000).

ESRI products of ArcInfo (for generating vector-based $V L I S$ ), and ArcView GIS (for generating various views/ scenarios) GIS software systems were used for the present studies.

\subsection{VLIS Development}

\subsubsection{Generation of Spatial Village Maps}

District and taluka boundaries were drawn from the SOI topographical maps. These were brought in as ArcInfo coverage after following the standard procedure: digitization, geo-referencing, etc. Taluka maps, collected from the local government offices in the district, contain village boundaries with contour lines and other topographical information. These taluka maps, which do not show geographical co-ordinates, were traced, marking Ground Control Points (GCPs) with respect to the SOI base map, digitized and brought to the real world coordinate system after projecting the maps (polyconic system) and carrying out the editing functions such as appending, edge-matching, etc. in Arc/Info. Each village in this map was assigned unique ids (user-defined) in a regular sequence. Thus, taluka map with village boundaries with in-built table having aerial extent, village-ids, etc., was generated for Thane district. The village map was opened in Arc/View for further processing.

\subsubsection{Transformation of Non-spatial Census Database into} GIS

1991 Census data collected from the NIC-Thane Office was a $d b f$ file. Census information can be displayed using the customized NIC software, called DISPLAN. This file contains vast information on ecological and socio-economic characteristics of each village, including census-codes on region, district, taluka and village. The $d b f$ file was later displayed in MS Excel sheets. Village-ids, same as given in the spatial village map, were fed in the Excel sheets for all the villages in the district. Thus, spatial and non-spatial data contain similar columns in their respective tables with uniform village ids (user-defined). These ids will help in joining the spatial and non-spatial data in the system.

\subsubsection{Integration of Spatial and Non-spatial Database}

The MS Excel file was converted into text format to enable opening in Arc/View. The Tables of both spatial village map and non-spatial census information were opened in Arc/View and joined together, with the help of user-defined ids, using table-join function.

Thus, an information system has been generated for the district showing the village map with its boundaries and the relevant census information containing ecological and socioeconomic dimensions. Generalized processing flow for developing VLIS model is depicted in Figure 1.

\subsubsection{Generation of Views for Decentralized Planning}

A few ecological and socio-economic views for identifying candidate villages for different rural watershed management schemes were generated for Dahanu sub-division of the district (containing Palghar, Dahanu and Talasari talukas):

(1) Total population (Figure 2a);

(2) SC/ST population (Figure 2b);

(3) Total geographical area (Figure 3a);

(4) Bank facilities (Figure 3b);

(5) Land utilization - Irrigated areas (Figure 4a);

(6) Land utilization - Net area sown (Figure 4b);

(7) Land utilization - forests (Figure 5a); 


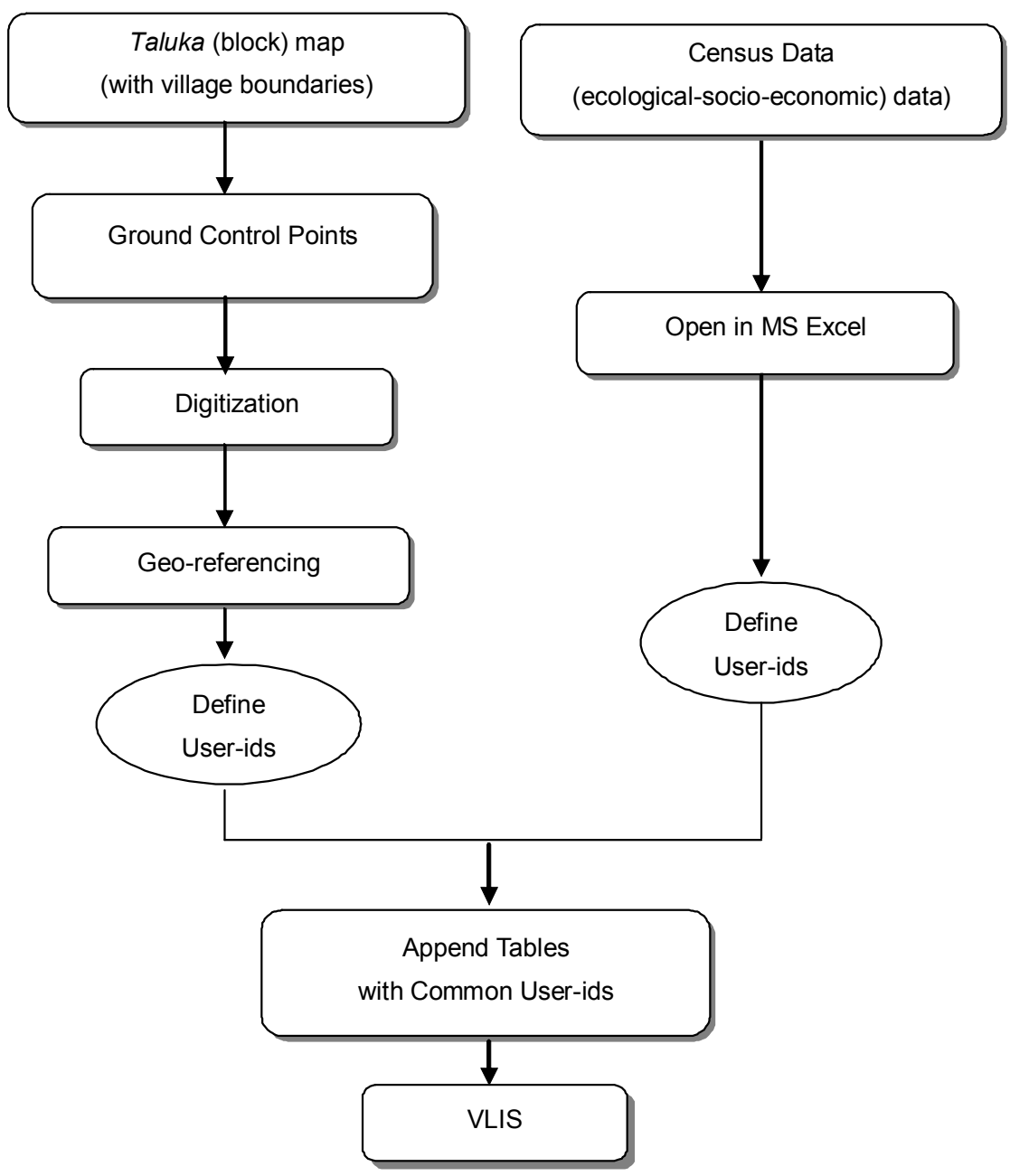

Figure 1. Generalized processing flow for Village Level Information System (VLIS).

(8) Land utilization - fallow lands (1 - 5 years) (Figure $5 b$ ).

Thematic mapping on available Census data for state and district level is possible with user defined class intervals or statistical intervals.

Likewise, the users can generate various views by themselves depending upon their needs, using the VLIS module, to make plans and take appropriate rural development decisions. For example, the scenarios/views on developing underprivileged SC/ST population village clusters (Figure $2 \mathrm{~b}$ ) would be useful in identifying hot spots for implementing the village-based poverty alleviation rural development schemes. However, VLIS is not useful in adjudging the candidate villages falling on the watershed boundary for watershed-based schemes. Presently, approximate proportionate values are taken in the adjoining villages of the watershed boundary. This has to be rectified in the future system. VLIS could be customized to give it a friendlier interface using the scripts available in Arc View.

After gaining access to the analyzed information, district staff may receive hands-on training in GIS and its userinterface, so that they will be better able to contribute their local and specialist knowledge to the development and application of the district database for decentralized planning.

This VLIS experience in the development of spatial information infrastructures provide a generic framework for districts facing a similar challenge.

\section{Conclusions}

A study has been undertaken in the tribal-oriented and rural-based Thane district in Maharashtra State, India to generate resource informatics to assist the decision-makers for decentralized planning with main emphasis on rural development. Major and significant conclusions emerging out of this 


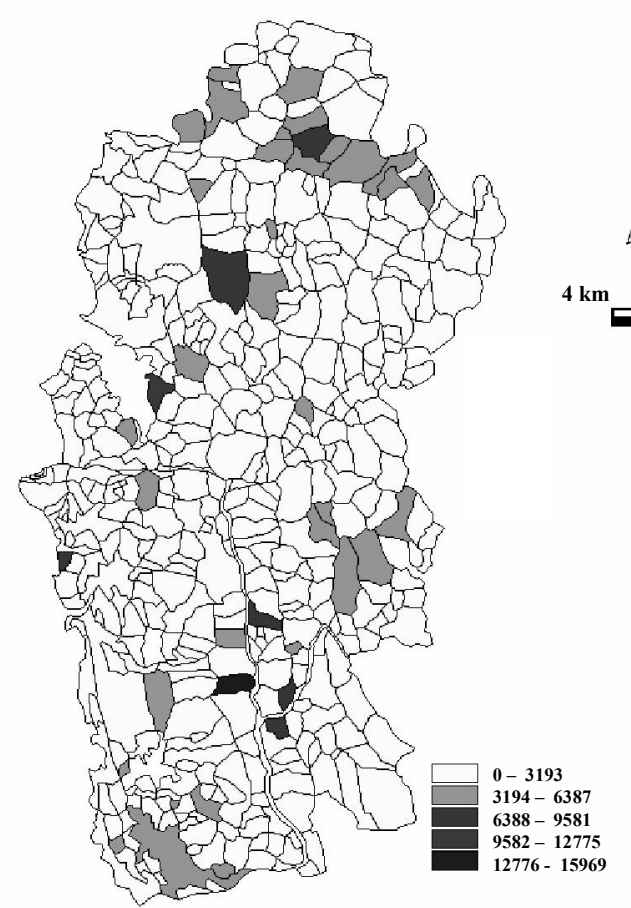

Figure 2a. Total population of Dahanu sub-division, Thane District, Maharashtra State, 1991.

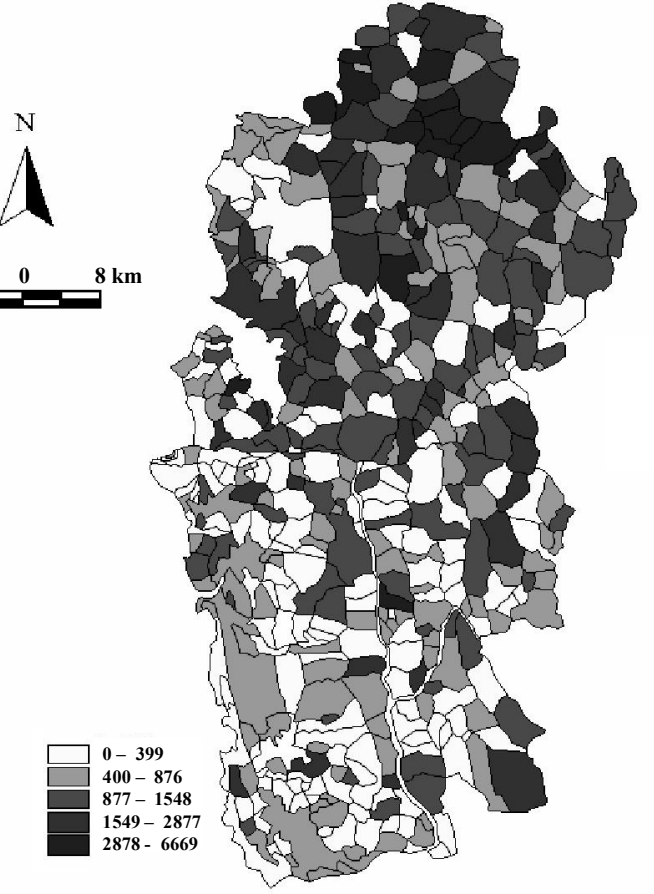

Figure 2b. SC/ST (under-privileged) population of Dahanu sub-division, Thane District, Maharashtra State, 1991.

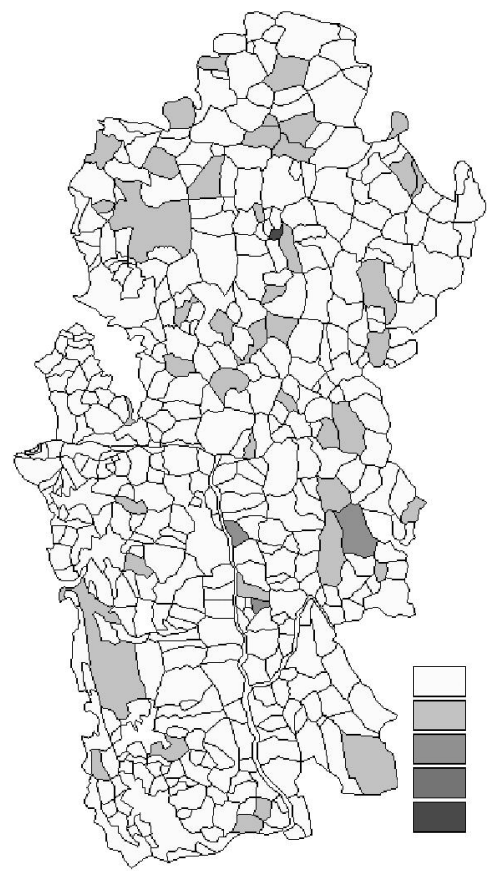

Figure 3a. Total geographical area of Dahanu sub-division, Thane District, Maharashtra State, 1991.

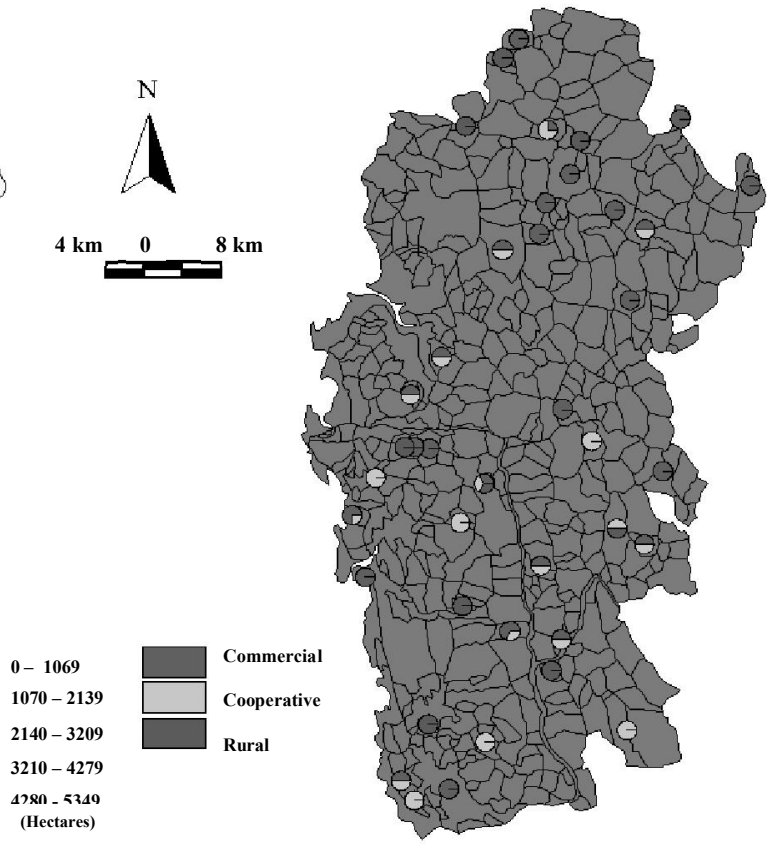

Figure 3b. Bank facilities of Dahanu sub-division, Thane District, Maharashtra State, 1991. 


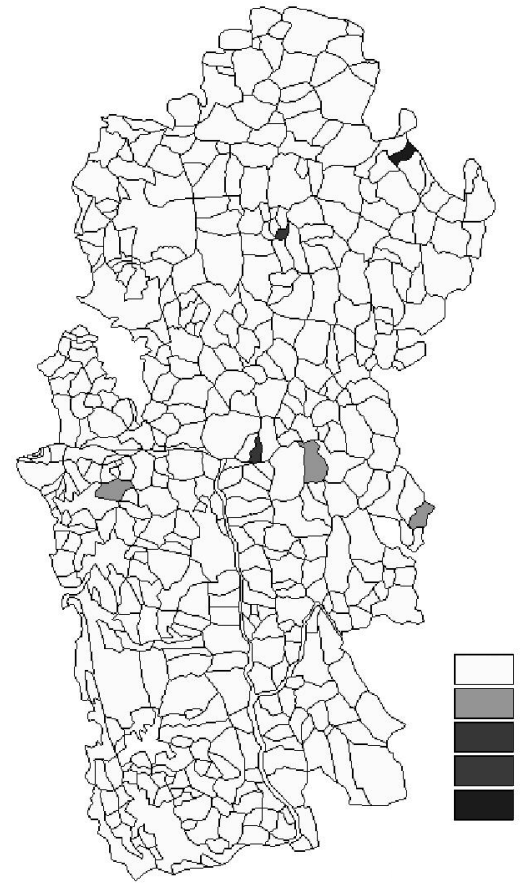

Figure 4a. Land utilization (irrigated areas) of Dahanu sub-division, Thane District, Maharashtra State, 1991.

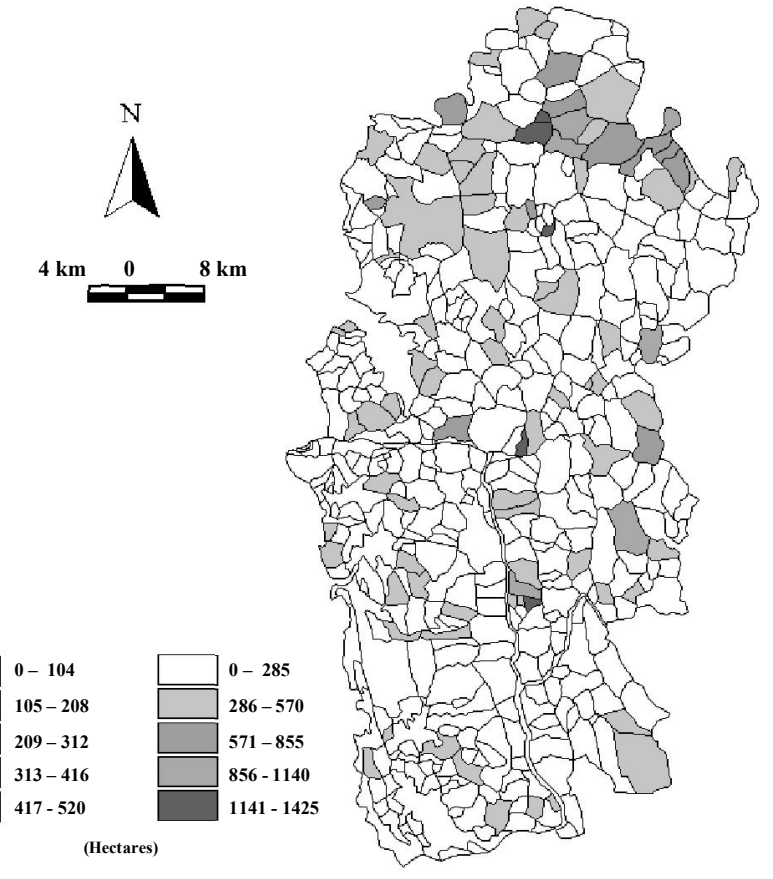

Figure 4b. Land utilization (net area sown) of Dahanu sub-division, Thane District, Maharashtra State, 1991.

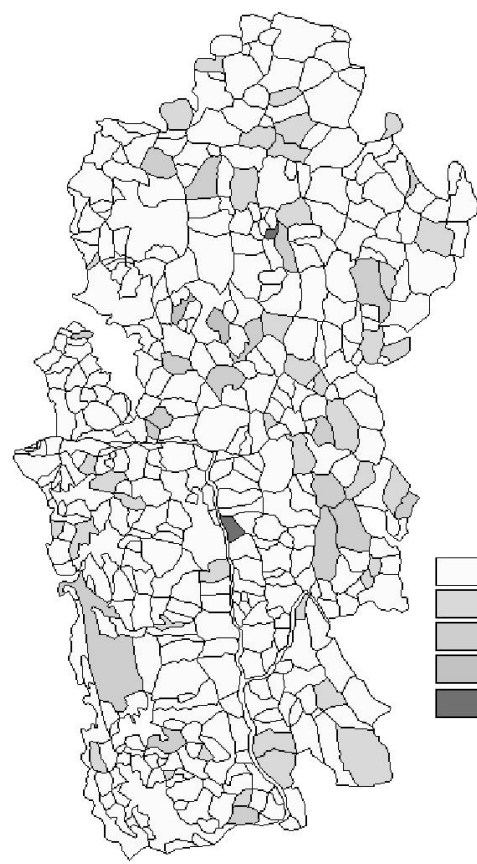

Figure 5a. Land utilization (forests) of Dahanu sub-division, Thane District, Maharashtra State, 1991.

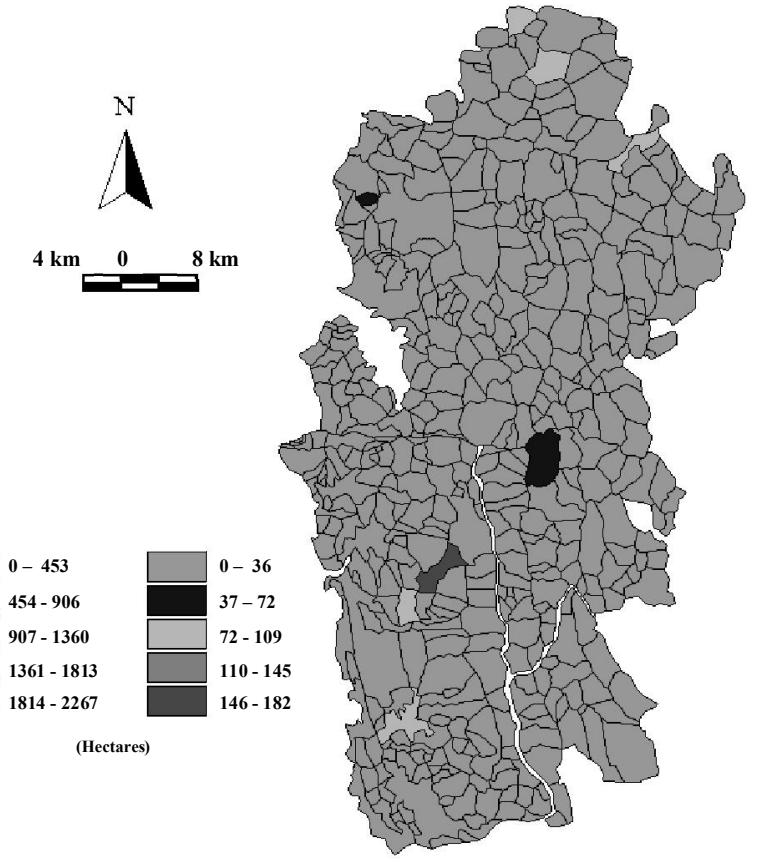

Figure 5b. Land utilization (fallow lands of 1-5 years) of Dahanu sub-division, Thane District, Maharashtra State, 1991. 
study are elucidated below:

VLIS tool, with a motto 'turning data into information', generated in the present study integrating the spatial village maps with non-spatial or tabular information from the NIC system, has demonstrated its potential for grass-root level development planning taking into consideration the local needs and constraints. It has also established its usefulness to the decision-makers in the district to generate views for decision-making at local-level. This prototype Community GIS tool will serve as a first step towards the development of Decision Support System for decentralized planning at district/sub-district level.

The main conclusion from this research is that the development of a digital base map as basic spatial infrastructure for a range of GIS rural applications is very difficult to achieve in the short to medium term. The main limitations are a lack of appreciation of what GIS can and cannot do, lack of resources and trained personnel, inefficient bureaucratic processes, lack of data, and lack of hardware and software vendor support.

In addition, it is suggested that small to medium scale GIS projects are the best option to introduce the GIS concept in the districts, to gain acceptance of the technology. In this regard, high resolution satellite imagery offers an opportunity to obtain the basic spatial data infrastructure or digital map base required to support small to medium scale rural GIS in future.

VLIS could have direct application in the districts, even though personnel, institutional and financial issues will continue to constrain adoption.

The GIS application for these individual projects may be relatively simple but may provide major benefits. For example, agriculture departments being the nodal agent for watershed management scheme implementation in the districts found villages showing percentage irrigated area, annual rainfall and under-privileged population, etc., is useful in identifying candidate villages for NWDPRA schemes.

\section{Future Developments}

Cheaper and faster Information and Communication Technology (ICT) is coming within the reach of most Organizations in the districts. It is therefore appropriate to consider the ways in which this technology has been implemented in the districts. In particular, future trends must be considered in order to understand the potential information infrastructure to which these districts may aspire - while recognizing that different problems and conditions exist in different districts.

With a better database, we can provide a better service to the user organizations to assist in their own decision-making process for developmental planning. A few possible future development programs for better service to the users for decentralized planning are:

(1) Customization: A better Graphical User Interface (GUI) could be built using GIS, as it provides very comprehensive and fast access to information, both graphically and non-graphically. This makes the system more robust in terms of its communication with a variety of users. (2) Strategic Unit for Decentralized planning: Given that all village informatics are now spatially part of a common coordinated system, a number of useful combinations can be performed. The first step in this process is to create Integrated Resource Unit (IRU). Each IRU comprises the spatial and non-spatial resource data, and can be taken as a strategic unit for assessing various decisions. Since they exhibit strong uniformity, they can all be expected to respond similarly to given intensities of human use and management strategies. Use of strategic units for treatment-oriented land use planning schemes for hilly watershed/terrain using GIS has been demonstrated in the studies carried out by Adinarayana and Rama Krishna (1995). (3) Decision Support System (DSS): As far as rural development planning is concerned, VLIS generated in the present study is unsophisticated but it is robust and functions with the data that are actually available in every NIC District Centre in India. This prototype VLIS could be customized to develop $D S S$ for decentralized planning, proposed to be called 'DecentPlan/DSS', to assist the decision-makers at district/sub-district level.

Some of the recommendations/future scenarios will be considered for execution in the second phase of the project, proposed with the Ministry of Rural Development, for a drought-prone district in India using the indigenously developed, low cost and simple Gram ++ (Geo-Referenced Area Management) (http://www.csre.iitb.ac.in/gram++/) GIS package which is available in many districts now for decentralized/rural development planning decisions.

Acknowledgments. This work forms part of the National Informatics Centre, Ministry of Communication and Information Technology, Government of India project 'GIS thematic mapping for decentralized planning at district/sub-district level'. Authors wish to thank NIC-Thane Centre for extending help and cooperation at various stages of the project. Thanks are also due to CSRE colleagues Drs. G. Venkataraman and M.V. Khire for useful help and discussions.

\section{References}

Adinarayana, J. and Rama Krishna, N. (1995). An approach to land-use planning in a hilly watershed using GIS. Land Degradation Rehabil., 6, 171-178.

Adinarayana, J., Maitra, S. and Dent, D. (2000). A spatial decision support system for land use planning at district level in India. The Land, 4(2), 111-130.

Anonymous (1995). Census of India, Thane District Census Handbook, Village \& Town Directory, Village \& Town wise Primary Census Abstract, Series 14, Maharashtra, Director of Census Operations, Mumbai, Maharashtra.

Bishop, I.D., Escobar, F.J., Kuruppannan, S., Williamson, I.P., Yates, P.M., Suwarnarat, K. and Yaqub, H.W. (2000). Spatial data infrastructures for cities in developing countries: Lessons from the Bangkok experience. Cities, 17 (2), 85-96.

Campbell, H. and Masser, I. (1995). GIS and Organizations, Taylor and Francis, London. 
Durga Rao, K.H.V., Aggarwal, S.P., Hari Prasad, V., Joshi, A. and Ray, P.S. (2002). Identification of suitable sites for watermills using remote sensing and GIS, (CD-ROM) Proceedings of the ISPRS Technical Commission VII Resource and Environmetal Monitoring Symposium, Hyderabad, India.

GRAM++: Geo-Referenced Area Management - A GIS tool. http://www.csre.iitb.ac.in/gram++.

Korte, G.B. (1994). The GIS Book, Onword Press, Santa Fe, CA.
NCGIA (1990). Core Curriculum. Vol. I: Introduction to GIS, Vol. II: Technical Issues in GIS, Vol. III: Application Issues in GIS, National Center for Geographic Information and Analysis, University of California, Santa Barbara, CA.

NIC (National Informatics Centre). Ministry of Communication and Information Technology, Government of India, New Delhi. http: //www.nic.in. 